\title{
Analysing Real-World Turbulence At Low Cost
}

\author{
N. P Mashaba and D. J Griffith
}

\begin{abstract}
Absorption, scattering and refractive index fluctuations are three primary phenomena contributing to optical turbulence. Tem- perature fluctuations, humidity and air velocity cause variations on both temporal and spatial, in the refractive index of the atmosphere, leading to beam wander, loss of coherence, beam directional fluctuations and irradiance fluctuations. Whereas, absorption and scattering affect the propagation of a laser beam as the constituent gases in the atmosphere interact with the beam resulting in an attenuation of the beam. Remarkably, this has led to the developing of systems, where coherent imaging can be tailored to demonstrate turbulence effects at low cost. We demonstrate the simulation of atmospheric turbulence in the laboratory using a digital micromirror device. We illustrate the advantages of this approach, as well as some of the limitations. We show experimental results demonstrating these limitations, and we discuss the impact they have on the simulation of various turbulence strengths.
\end{abstract}

Keywords - Digital micromirrors, laser beams, turbulence, Zernike polynomials

\section{INTRODUCTION}

When a laser beam propagates through the atmosphere it experi- ences turbulence. The result of these atmospheric turbulence effects can be random beam variations which can be deleterious on the beam quality of a laser beam (see Fig. 1). The randomness in the atmosphere is caused by the difference in the refractive index of air. Various densities of random air mix, resulting random refractive index in the atmosphere. This mixing of the refractive index of air operatesin convective currents that move air packets of varying size around. The inner scale (smaller scale) of turbulent, below which viscous effects are important, while the largest eddies define the so-called 'outer scale', above which the atmosphere is considered non-isotropic. Kolmogorov assumes the simplified problem of a non-viscous and isotropic atmosphere, so that the inner scale, 10 becomes zero and the outer scale, L0 is infinity. These presumptions can be directly applied in the laboratory, giving a good approximation for a real atmosphere [1] via a well-defined distribution of the random refractive index of the atmosphere.

Here we demonstrate a low-cost technique that employ modern digital micromirror devices (DMDs). We analyse atmospheric turbulence for better vision in astronomy and

Manuscript received 10 June, 2020

This work was supported by the Optronics Sensor Systems (OSS) group at the CSIR.

N. P Mashaba is with Optronics Sensor Systems department at The Council for Scientific and Industrial Research (CSIR), Defence and Security Cluster, P. O. Box 395, Pretoria, South Africa,

D. J Griffith is with Hensoldt Optronics, Nellmapius Dr, Highveld, Centurion, 0157. military applications. A DMD is an electronic device that have an ability to control the amplitude of an incoming light into to a desired field. DMDs can also control the phase and amplitude of light through complex-amplitude modulation [2]. We will review the theory needed to create simple turbulence phase screens, and then show how to implement these in a laboratory (lab). Lastly, we will show how to quantify turbulence on an imaging system in the lab.

\section{ZERNIKE POLYNOMIALS}

Over the past years, Zernike polynomials have been utilised in a myriad of experiments for creating turbulence phase screens to study the effects of turbulence on laser beams [3-5]. This numerical technique approximates the phase that can distort a laser beam. The method is chosen to simulate turbulence phase screens because it forms a neat basis-set and it is orthogonal over a unit circle [6].

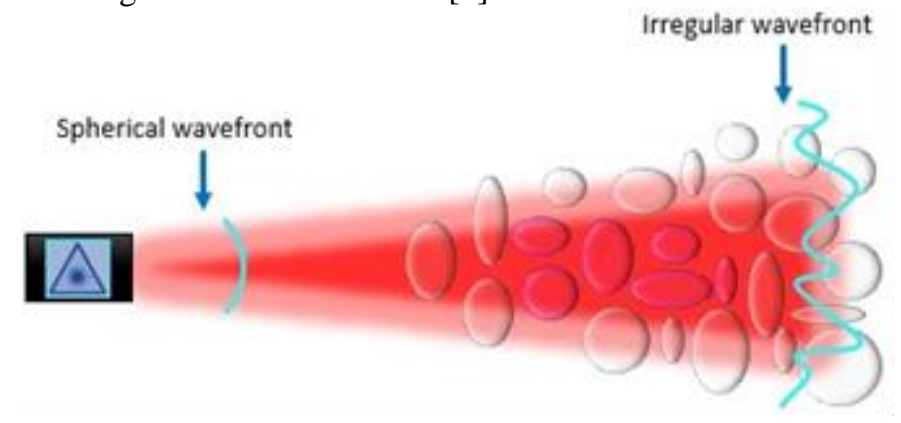

Fig. 1. A spherical wavefront propagating through a turbulent atmosphere. The resulting field is a distorted wavefron

\begin{tabular}{ccccc}
$j$ & $n$ & $m$ & $Z_{j}(\rho, \theta)$ & Aberration \\
1 & 0 & 0 & 1 & Piston \\
2 & 1 & 1 & $2 \rho \cos \theta$ & x tilt \\
3 & 1 & 1 & $2 \rho \sin \theta$ & y tilt \\
4 & 2 & 0 & $\sqrt{3}\left(2 \rho^{2}-1\right)$ & Defocus \\
5 & 2 & 2 & $\sqrt{6} \rho^{2} \sin 2 \theta$ & 45 deg Primary astigmatism \\
6 & 2 & 2 & $\sqrt{6} \rho^{2} \cos 2 \theta$ & 0 deg Primary astigmatism \\
7 & 3 & 1 & $\sqrt{8}\left(3 \rho^{3}-2 \rho\right) \sin \theta$ & Primary y coma \\
8 & 3 & 1 & $\sqrt{8}\left(3 \rho^{3}-2 \rho\right) \cos \theta$ & Primary x coma \\
9 & 3 & 3 & $\sqrt{8} \rho^{3} \sin 3 \theta$ & \\
10 & 3 & 3 & $\sqrt{8} \rho^{3} \cos 3 \theta$ \\
11 & 4 & 0 & $\sqrt{5}\left(6 \rho^{4}-6 \rho^{2}+1\right)$ & TABLE I \\
\multicolumn{5}{c}{ ZERNIKE POLYNOMIALS, ZJ WITH AZIMUTHAL spherical AND RADIAL } \\
\multicolumn{5}{c}{ INDICES, N AND M [7] }
\end{tabular}

The Zernike polynomials can be can be calculated from [811]

$$
Z_{j}(\rho, \theta)=\left\{\begin{array}{lll}
U_{n m}(\rho, \theta) & : m<0 ; & |m-n|=\text { even } \\
V_{n m}(\rho, \theta) & : m \neq 0 ; & |m-n|=\text { odd } \\
R_{n}^{0}(\rho) & : m=0
\end{array}\right.
$$


where the parameters

While

$$
\begin{aligned}
& U_{n m}(\rho, \theta)=R_{n}^{m}(\rho) \cos m \theta, \\
& V_{n m}(\rho, \theta)=R_{n}^{m}(\rho) \sin m \theta,
\end{aligned}
$$

$$
R_{n}^{m} \rho=\sum_{s=0}^{(n-m) / 2} \frac{(-1)^{s}(n-s !)}{s !\left(\frac{n+m}{2}-s\right) !\left(\frac{n-m}{2}-s\right) !} \rho^{n-2 s}
$$

Here the radial and azimuthal part are presented as $\mathrm{n}$ and $\mathrm{m}$. The phase function, $(\varphi=(\rho, \theta))$ of a turbulence phase screen is given by

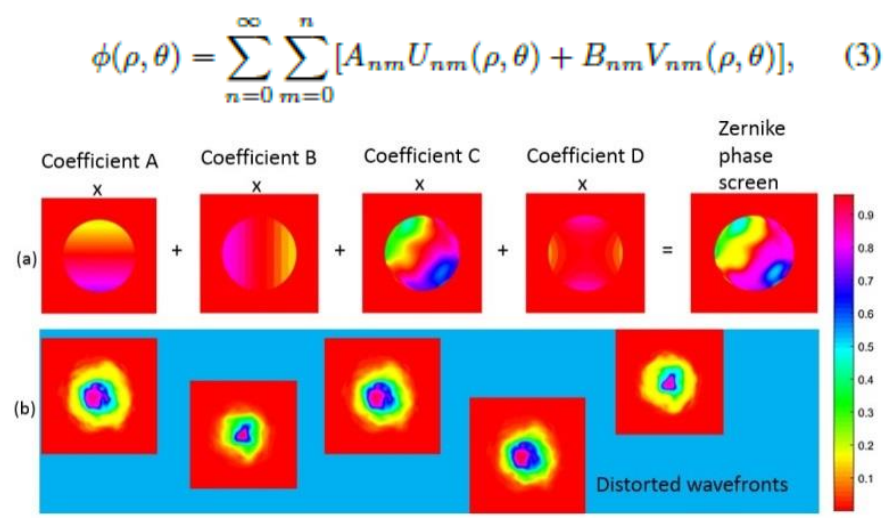

Fig. 2. (a) Sum of Zernike polynomials, $\mathrm{Zj}$ multiplied by their coefficient that can also be presented as aberrations. Here coefficient A, B, C and D are $\mathrm{x}$-tilt, $\mathrm{y}$-tilt, defocus, and astigmatism from Table. I. (b) Distorted wavefront intensity profiles, resulting from an aberrated field.

where the coefficients

$$
\begin{aligned}
& A_{n m}=K(m)\left(\frac{n+1}{\pi}\right) \int_{0}^{2 \pi} \int_{0}^{1} \phi(\rho, \theta) U_{n m}(\rho, \theta) \rho \mathrm{d} \rho \mathrm{d} \theta, \\
& B_{n m}=K(m)\left(\frac{n+1}{\pi}\right) \int_{0}^{2 \pi} \int_{0}^{1} \phi(\rho, \theta) V_{n m}(\rho, \theta) \rho \mathrm{d} \rho \mathrm{d} \theta
\end{aligned}
$$

And

$$
K(m)=\left\{\begin{array}{cc}
2: & \text { for } m=0, n \neq 0 \\
1: & \text { otherwise }
\end{array} .\right.
$$

To compute the amplitudes, $A_{n m}$ and $B_{n m}$, we sample the values with a normal distribution with zero mean and a variance to make the phase screens realistic. The variance is given by

$$
\sigma_{n m}^{2}=I_{n m}\left(D / r_{0}\right)^{5 / 3},
$$

where $\mathrm{D}$ is the aperture size of the imaging system, while the coherence length is

$$
r_{0}=1.68\left(C_{n}^{2} L k^{2}\right)^{-3 / 5} .
$$

The $\mathrm{r}_{0}$ in an infinite space can also be written as

$$
r_{0}=\left(0.423 k^{2} \int_{0}^{L} C_{n}^{2}(z) d z\right)^{-3 / 5},
$$

While

$$
I_{n m}=\frac{0.15337(-1)^{n-m}(n+1) \Gamma(14 / 3) \Gamma(n-5 / 6)}{\Gamma(17 / 6)^{2} \Gamma(n+23 / 6)} .
$$

Herein the $\Gamma(\bullet)$ is the gamma function.

\section{IN THE LAB}

To calculate the real-world phase screens we used $\mathrm{C}_{\mathrm{n}}^{2}$ values, acquired by a scintillometer. A scintillometer is an instrument that measures the turbulence strength, $\mathrm{C}_{\mathrm{n}}^{2}$ in the field. Figure 2 (a) shows a turbulence phase screen that we generated using Eq. (3). Once the phase screens were computed, we then generated holograms that represent these turbulence phase functions onto a DMD. The turbulence hologram can be written as [2]

$$
\begin{aligned}
H(x, y)= & \frac{1}{2}+\operatorname{sign}\left(\cos \left[n_{x}+n_{y}+\pi p(x, y)\right]\right. \\
& +\cos (\pi w(x, y))) .
\end{aligned}
$$

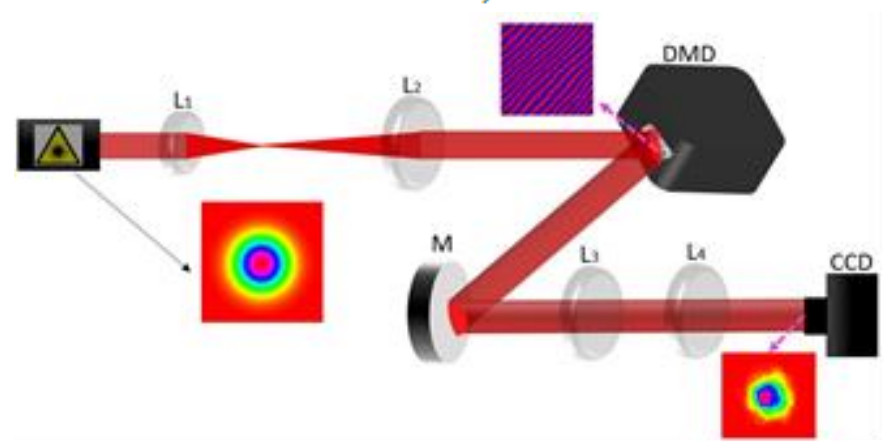

Fig. 3. A collimated and expanded (using lenses $\mathrm{L}_{1}=50 \mathrm{~mm}$ and $\mathrm{L}_{2}=$ $100 \mathrm{~mm}$ ) helium neon laser illuminating a turbulence hologram onto the DMD. A 4-f system (lenses $\mathrm{L}_{3}=\mathrm{L}_{4}=100 \mathrm{~mm}$ ) was added to image the plane of the DMD. A final distorted beam is observed.

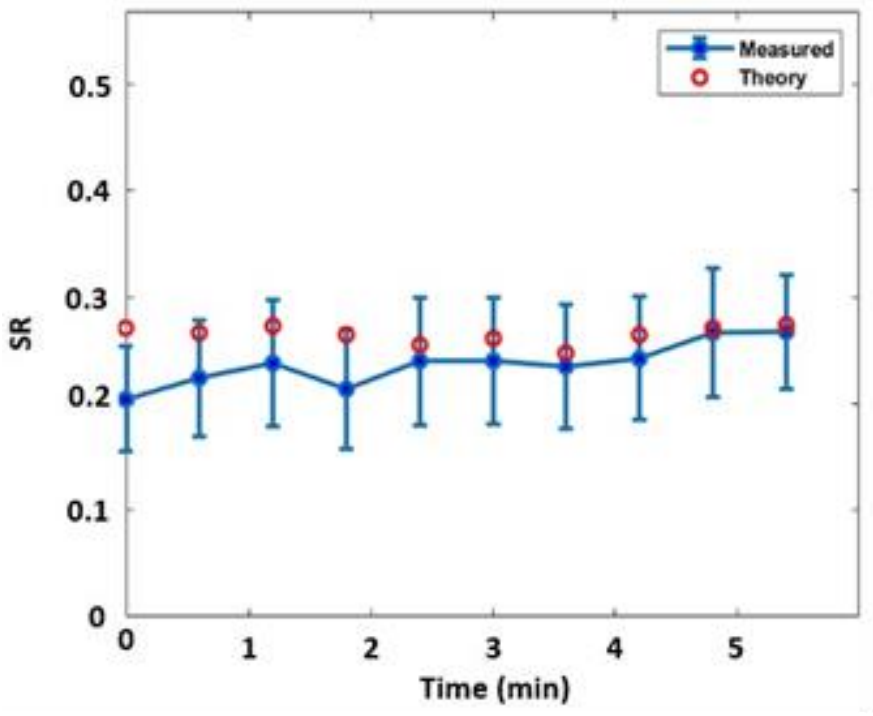

Fig. 4. Strehl Ratio vs time. The Strehl ratio measures turbulence strength in a scale of zero (strong turbulence) upto 1 (being weak turbulence).

Here $w(x, y)=(1 / \pi) \times \arcsin (A(x, y))$ and $p(x, y)=(1 / \pi) \times$ $\psi(\mathrm{x}, \mathrm{y})$ equivalent to the amplitude and phase of light. $\psi(\mathrm{x}, \mathrm{y})$ is the phase function of the turbulence phase screens. An example of a hologram (stripes) is shown in Fig. 3.

To analyse turbulence strength on an optical system, we measured the Strehl Ratio in the lab. The experimental form of SR can be written as $S R=I_{\text {turb }}(0,0) / I(0,0)$ which represents the ratio of the central peak for an unperturbed beam and the perturbed beam. We mainly compared the on-axis intensity of the turbulent with a non-turbulent Gaussian beam using

$$
\mathrm{SR}=\frac{I(0)}{I_{0}(0)} \approx \frac{1}{\left[1+\left(D / r_{0}\right)^{5 / 3}\right]^{6 / 5}} .
$$


I (0) and $\mathrm{I}_{0}(0)$ are the on-axis intensities of the distorted wavefront and initial intensity of the Gaussian beam. To do this in the lab, we illuminated the DMD with an expanded and collimated Helium Neon laser of 632.8 nanometers wavelength. A first order was filtered using a pinhole and it was imaged at the focal plane of the second lens $\left(\mathrm{L}_{4}\right)$ of the 4-f system. Here, the turbulence holograms were loaded on the DMD at a very fast rate that looked realistic. Fig. 2 (b) show the resulting Gaussian beam after passing the perturbed beam. And the quantitative (Strehl Ratio) results of this beams are shown in Fig 4. We recorded the on-axis intensity for each image. The SR was calculated from these intensities (turbulence and non-turbulent) and then compared them to the theory. The results indicate strong turbulence and both the theory and experimental results agrees.

The main limitation of using this approach to analyse real-world turbulence is that, this measurements can only be done in the laboratory and not outdoor because of the nature of optics. Stray light will interfere with the beam and result in false analyses. In future, we are planning on building a system that can automatically analyse and correct real-world turbulence in real-time.

\section{CONCLUSION}

In this work we have demonstrated the simulation of atmospheric turbulence in the laboratory using cheap optics and modern digital micromirror devices. We illustrated the advantages of this approach, as well as some of the limitations.

\section{REFERENCES}

[1] L. C. Andrews and R. L. Phillips, Laser beam propagation through random media, vol. 152. SPIE press Bellingham, WA, 2005. https://doi.org/10.1117/3.626196

[2] M. Mirhosseini, O. S. Magana-Loaiza, C. Chen, B. Rodenburg, M. Malik, and R. W. Boyd, "Rapid generation of light beams carrying orbital angular momentum," Optics express, vol. 21, no. 25, pp. 30196-30203, 2013.

https://doi.org/10.1364/OE.21.030196

[3] M. Malik, M. O’Sullivan, B. Rodenburg, M. Mirhosseini, J. Leach, M. P. Lavery, M. J. Padgett, and R. W. Boyd, "Influence of atmospheric turbulence on optical communications using orbital angular momentum for encoding," Optics express, vol. 20, no. 12, pp. 13195-13200, 2012. https://doi.org/10.1364/OE.20.013195

[4] S. Zhao, J. Leach, L. Gong, J. Ding, and B. Zheng, "Aberration corrections for free-space optical communications in atmosphere turbulence using orbital angular momentum states," Optics express, vol. 20, no. 1, pp. 452- 461, 2012. https://doi.org/10.1364/OE.20.000452

[5] M. Cheng, L. Guo, J. Li, and Y. Zhang, "Channel capacity of the oambased free-space optical communication links with bessel-gauss beams in turbulent ocean," IEEE Photonics Journal, vol. 8, no. 1, pp. 1-11, 2016. https://doi.org/10.1109/JPHOT.2016.2518865

[6] R. J. Noll, "Zernike polynomials and atmospheric turbulence," JOsA, vol. 66, no. 3, pp. 207-211, 1976

[7] R. J. Noll, "Zernike polynomials and atmospheric turbulence," Journal of the Optical Society of America, vol. 66, p. 207, 31976. https://doi.org/10.1364/JOSA.66.000207

[8] R. J. Noll, "Zernike polynomials and atmospheric turbulence," JOsA, vol. 66, no. 3, pp. 207-211, 1976. https://doi.org/10.1364/JOSA.66.000207

[9] V. N. Mahajan and G.-m. Dai, "Orthonormal polynomials in wavefront analysis: analytical solution," JOSA A, vol. 24, no. 9, pp. 2994-3016, 2007.

https://doi.org/10.1364/JOSAA.24.002994
[10] V. N. Mahajan and J. A. D'1az, "Imaging characteristics of zernike and annular polynomial aberrations," Applied optics, vol. 52, no. 10, pp. 2062 $-2074,2013$ https://doi.org/10.1364/AO.52.002062

[11] V. N. Mahajan, "Optical imaging and aberrations(part ii, wave diffraction optics)," SPIE- The International Society for Optical Engineering, 1998. 